\title{
Velocity Control with Gravity Compensation for Magnetic Helical Microswimmers
}

\author{
Arthur W. Mahoney ${ }^{\text {a,* }}$, John C. Sarrazin ${ }^{\mathrm{b}}$, Eberhard Bamberg ${ }^{\mathrm{b}}$ and Jake J. Abbott $^{\mathrm{b}}$ \\ ${ }^{a}$ School of Computing, University of Utah, Salt Lake City, UT 84112, USA \\ ${ }^{\mathrm{b}}$ Department of Mechanical Engineering, University of Utah, Salt Lake City, UT 84112, USA
}

Received 25 June 2010; accepted 11 August 2011

\begin{abstract}
Magnetic helical microswimmers, which swim using a method inspired by the propulsion of bacterial flagella, are promising for use as untethered micromanipulators and as medical microrobots. Man-made devices are typically heavier than their fluid environment and consequently sink due to their own weight. To date, methods to compensate for gravitational effects have been ad hoc. In this paper, we present an open-loop algorithm for velocity control with gravity compensation for magnetic helical microswimmers that enables a human operator or automated controller to command desired velocity intuitively, rather than directly controlling the microswimmer's orientation and rotation speed. We provide experimental verification of the method.
\end{abstract}

(c) Koninklijke Brill NV, Leiden and The Robotics Society of Japan, 2011

Keywords

Microrobot, helix, screw, magnetic, wireless, untethered

\section{Introduction}

Untethered biomedical microrobots are a topic of considerable research. Microrobots have the potential to radically transform many medical procedures by operating in hard-to-reach locations of the body, performing tasks such as targeted therapy (e.g., drug delivery, hyperthermia), tissue removal (e.g., ablation) and remote sensing, and the bodies of the microrobots themselves may be used as maneuverable structures (e.g., stents, tissue scaffolding) [1]. Microrobots have also already been demonstrated as useful tools for untethered manipulation at the microscale, both on planar surfaces $[2,3]$ and in three-dimensional fluidic environments $[4,5]$. Un-

\footnotetext{
* To whom correspondence should be addressed. E-mail: art.mahoney@utah.edu
} 
tethered microrobots stand to impact a broad range of fields, but we must first understand their locomotion and how to effectively control them wirelessly.

The environment in which a microrobot operates is significantly different than that of macroscale robots as we know them. In particular, microrobots typically operate in fluids at the low-Reynolds-number regime, where viscous drag significantly dominates over inertia [6]. In this environment, propulsion and actuation can be challenging, and although there are a number of ways for a microrobot to be wirelessly propelled, one method of particular interest is helical propulsion a swimming method inspired by the propulsion of bacterial flagella - using torque produced from the rotation of an externally applied magnetic field [4, 7, 8]. It has been shown that magnetic helical swimming performs well compared to other methods of magnetic propulsion as the size of the microrobot scales down [9]. Microrobots that are propelled in this manner are typically simple devices consisting of a magnetic body rigidly attached to a chiral structure resembling a cork screw or wood screw. The community now has a good basic understanding of the ways in which magnetic helical microswimmers can be controlled and researchers have begun to tackle some of the non-idealities that manifest themselves during experimentation [10].

One such non-ideality is the microrobot sinking due to its own weight. Magnetic control of helical microswimmers has typically started from the assumption that the microswimmers behave similarly to bacteria, which are approximately neutrally buoyant, and the microswimmer is simply pointed in the direction of desired velocity, as depicted in Fig. 1a. When swimming under an optical microscope, the microswimmer typically lies near the horizontal plane, such that it can be easily observed and controlled. However, man-made microswimmers are typically heavier than their fluid medium and they sink down, albeit slowly, due to their own weight, resulting in a velocity that is not aligned with the thrust direction of the microswimmer (Fig. 1b and d), thus making the microswimmer attain a desired velocity is not as simple as merely pointing the microrobot in the desired direction. To counteract this downward drift and keep the microswimmer at a desired focal plane, researchers have pitched the microswimmer up in an ad hoc manner, as shown in Fig. 1c, with the goal of keeping the long axis of the microswimmer close to the horizontal plane such that observation through the microscope is relatively unchanged. In Ref. [4], for example, the authors report that their microswimmers require a compensation pitch of approximately $10^{\circ}-20^{\circ}$, with the necessary pitch being inversely proportional to the microswimmer's rotation speed. As we will show herein, there is a unique combination of pitch angle and rotation speed (modulo $180^{\circ}$ change in pitch) to achieve the desired velocity of the microswimmer, whether or not the desired velocity lies in the horizontal plane, and this combination of pitch angle and rotation speed is not intuitive to a human operator. In this paper, we describe a velocity-control-plus-gravity-compensation algorithm that calculates the correct pitch angle and rotation speed to achieve the commanded velocity with results like those shown in Fig. 1e, making teleoperation much more intuitive. 


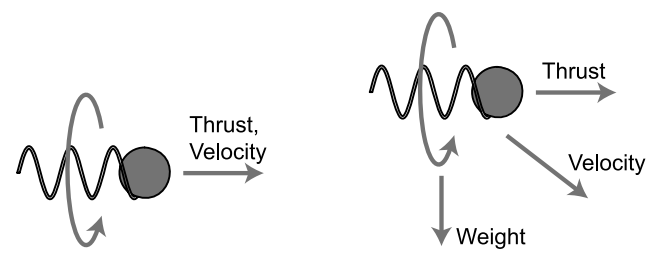

(a) Neutrally buoyant swimmer (b) Heavy swimmer

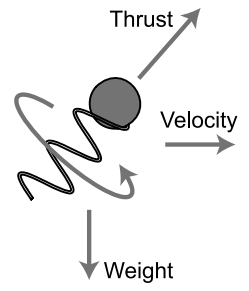

(c) Gravity compensation

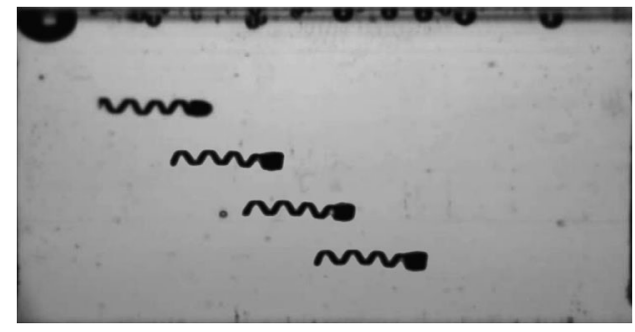

(d) Experiment without gravity compensation

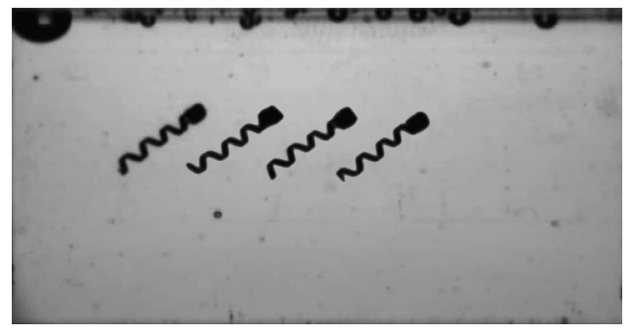

(e) Experiment with gravity compensation

Figure 1. (a) Natural flagellated bacteria are nearly neutrally buoyant. (b) Man-made swimmers are denser than water, causing them to drift downward under their weight. (c) To compensate for this drift, the microswimmer must be commanded to swim at a unique pitch angle and rotation speed. ( $d$ and e) Composite images from scaled experiments where the swimmer is commanded to move horizontally with a constant velocity, without and with the proposed gravity-compensation algorithm, demonstrating the behaviors described in (b) and (c), respectively. Gravity points downward in the images.

Considering the command input of magnetic helical microswimmer to be spatial velocity rather than manual control of pitch and rotation speed has not been done previously. It may be more appropriate to think of microswimmers as tiny helicopters as opposed to bacteria. Rather than the default configuration of the microswimmer being in the horizontal plane, it should be pointed vertically such that the thrust of the microswimmer is directly counteracting gravity. From this vertical configuration, the microrobot can move up or down simply by changing the rotation speed. To make lateral movements, the microswimmer pitches away from vertical, and again there is a unique combination of pitch angle and rotation speed to achieve the desired velocity.

To accurately command a velocity to our microswimmer that is not aligned with the microswimmer's central axis requires a model of the microswimmer that goes beyond the simple 2-d.o.f. model that has been used in prior work; in Section 2 we develop a full 6-d.o.f. model for helical microswimmers. In Section 3 we develop an algorithm that calculates the microswimmer orientation and rotation speed needed to achieve a desired spatial velocity. We experimentally verify the algorithm in Section 4, using a scaled magnetic swimmer propelled by the rotating uniform magnetic field of an electromagnetic system consisting of nested Helmholtz coils. Finally, in Section 5 we provide some additional discussion and interpretation of our algorithm and experimental results. 


\section{The 6-d.o.f. Helical Microswimmer Model}

The swimming properties of magnetic helical microswimmers are well understood for motion along the microswimmer's central axis $[6,9,11]$. In a low-Reynoldsnumber regime, the applied nonfluidic torque and force, $\tau$ and $f$, acting along the microswimmer's central axis are linearly related to the translational velocity and angular velocity, $v$ and $\omega$, also along the microswimmer's central axis. This is expressed in the form of the widely used system of equations:

$$
\left[\begin{array}{l}
f \\
\tau
\end{array}\right]=\left[\begin{array}{ll}
a & b \\
b & c
\end{array}\right]\left[\begin{array}{c}
v \\
\omega
\end{array}\right],
$$

where $a, b$ and $c$ are scalars that depend upon the geometry of the microswimmer and the properties of the fluid in which it swims. This 2-d.o.f. axial model has been used with good success to study the feasibility of helical swimming $[9,11]$ and to characterize basic swimming properties [12]; however, it does not provide sufficient information to understand how a helical microswimmer behaves in full 6-d.o.f. motion.

In this section, we extend (1) from 2 to 6 d.o.f. using resistive force theory (RFT) and spatial transformations, including effects caused by rigidly fixing a spherical magnetic head to the end of a helix. Making the assumption that the fluidic forces acting on the helix and the head are independent from each other, we first determine the fluidic force and torque acting purely on the helix, $\mathbf{f}_{\mathrm{h}}$ and $\boldsymbol{\tau}_{\mathrm{h}}$, using RFT, and then the fluidic force and torque acting solely on the spherical magnet head, $\mathbf{f}_{\mathrm{m}}$ and $\tau_{\mathrm{m}}$ (this assumption has been used in other work to obtain a good approximations of the fluidic properties of rigid helices in the low Reynolds regime $[9,13])$. Thus, the total force and torque acting on the microswimmer is the sum of the forces and torques acting on the helix and the spherical head: $\mathbf{f}=\mathbf{f}_{\mathrm{h}}+\mathbf{f}_{\mathrm{m}}$ and $\boldsymbol{\tau}=\boldsymbol{\tau}_{\mathrm{h}}+\boldsymbol{\tau}_{\mathrm{m}}$, respectively. Although we only consider helices of right-handed chirality, the results derived in this section can be obtained for left-handed helices using the same methods. A similar 6-d.o.f. model was presented in Ref. [13] for the study of cellular flagella motility where the helical flagella rotates opposite the direction of the cell body.

RFT is a simple approach used to determine the forces caused by velocity on an infinitesimally small length of helix $[14,15]$. RFT takes the velocity $\mathbf{v}_{s}$ of one of these small segments of the helix, decomposes the velocity into components parallel and perpendicular to the segment, and relates them to parallel and perpendicular drag forces acting on the segment with the differential forces:

$$
\begin{aligned}
\mathrm{d} \mathbf{f}_{\perp s} & =\xi_{\perp} \mathbf{v}_{\perp s} \mathrm{~d} s \\
\mathrm{~d} \mathbf{f}_{\| s} & =\xi_{\|} \mathbf{v}_{\| s} \mathrm{~d} s,
\end{aligned}
$$

where $\xi_{\perp}$ and $\xi_{\|}$are scalar drag coefficients, which have a number of empirical approximations (e.g., Ref. [15]). Integrating these differential forces along the length of the helix provides the fluidic forces acting on the helix $\left(\mathbf{f}_{\mathrm{h}}\right)$ induced by a spe- 


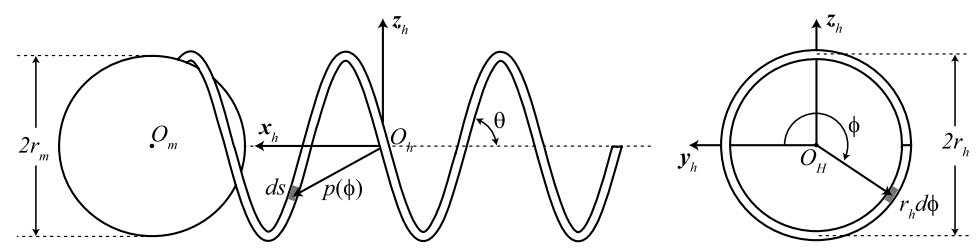

Figure 2. A helical microswimmer consisting of spherical magnetic body of radius $r_{\mathrm{m}}$ attached to a right-handed helix with pitch $\theta$ and radius $r_{\mathrm{h}}$. The coordinate frame of the microswimmer's helix (denoted by h) is oriented with $\mathbf{x}_{\mathrm{h}}$ along the central axis of the helix with the origin $\mathbf{O}_{\mathrm{h}}$ of the right-handed coordinate system at the center of the helix.

cific movement of the helix. To perform this integration in three dimensions, we first need to define the coordinate frames for the helix and for a given differential segment. Let the geometry of the helix be defined with pitch $\theta$ and radius $r_{\mathrm{h}}$, and let the helix coordinate frame (denoted by $\mathrm{h}$ ) lie with the $\mathbf{x}_{\mathrm{h}}$ axis parallel to the central axis of the helix, and the $\mathbf{y}_{\mathrm{h}}$ and $\mathbf{z}_{\mathrm{h}}$ axis be arranged as in Fig. 2. The origin of this coordinate system is located at the center of the helix, denoted by $\mathbf{O}_{\mathrm{h}}$. For ease of integration, we will describe the helix using a cylindrical coordinate system parameterized by the polar angle $\phi$. The matrix that will rotate vectors written in a given segment coordinate frame (denoted by s) to the helix frame is ${ }^{\mathrm{h}} \mathbf{R}_{\mathrm{s}}(\phi)=\mathbf{R}_{x}(\phi) \mathbf{R}_{y}(-\theta)$, where ${ }^{\mathrm{h}} \mathbf{R}_{\mathrm{s}} \in S O(3)$ denotes a rotation matrix from frame s to frame $\mathrm{h}$, and $\mathbf{R}_{x}(\phi) \in S O(3)$ denotes a rotation matrix about axis $\mathbf{x}$ by $\phi \operatorname{rad~[16].~We~can~write~the~vector~from~} \mathbf{O}_{\mathrm{h}}$ to the origin of each segment frame with respect to $\phi$ in the form:

$$
{ }^{\mathrm{h}} \mathbf{p}(\phi)=\left[\begin{array}{lll}
\frac{r \phi}{\tan (\theta)} & r \cos (\phi) & r \sin (\phi)
\end{array}\right]^{\mathrm{T}},
$$

where ${ }^{\mathrm{h}} \mathbf{p}$ denotes the vector $\mathbf{p}$ represented in frame $\mathrm{h}$. At each of these points, the $\mathbf{x}_{\mathrm{S}}$ axis of the segment frame lies parallel to the helix segment, and both $\mathbf{y}_{\mathrm{s}}$ and $\mathbf{z}_{\mathrm{S}}$ lie perpendicular to the helix segment, with $\mathbf{y}_{\mathrm{s}}$ pointing radially away from the central axis of the microswimmer's helix. This leads us to express the differential relating velocity to force in three dimensions with respect to the frame of an arbitrary segment along the helix in the segment frame as:

$$
{ }^{\mathrm{s}} \mathrm{d} \mathbf{f}_{s}={ }^{\mathrm{s}} \boldsymbol{\Xi}^{\mathrm{s}} \mathbf{v}_{s} \mathrm{~d} s
$$

where:

$$
{ }^{\mathrm{s}} \boldsymbol{\Xi}=\left[\begin{array}{ccc}
\xi_{\|} & 0 & 0 \\
0 & \xi_{\perp} & 0 \\
0 & 0 & \xi_{\perp}
\end{array}\right],
$$

and ${ }^{\mathrm{s}} \mathbf{f}_{s}$ and ${ }^{\mathrm{s}} \mathbf{v}_{s}$ are the force and velocity of the segment expressed in the segment's own frame. The relationship between velocity and forces in the segment frame can be expressed in the helix frame as:

$$
\mathrm{h}_{\mathrm{d}} \mathbf{f}_{s}={ }^{\mathrm{h}} \boldsymbol{\Xi}(\phi)^{\mathrm{h}} \mathbf{v}_{s} \mathrm{~d} s,
$$


where:

$$
{ }^{\mathrm{h}} \boldsymbol{\Xi}(\phi)={ }^{\mathrm{h}} \mathbf{R}_{\mathrm{s}}(\phi)^{\mathrm{s}} \boldsymbol{\Xi}^{\mathrm{s}} \mathbf{R}_{\mathrm{h}}(\phi) .
$$

As the helix is swimming, the velocity $\mathbf{v}_{s}$ of an infinitesimally small segment of helix (parameterized by $\phi$ with position described by $\mathbf{p}(\phi)$ ) can be decomposed into the sum of the microswimmer's translational velocity $\mathbf{v}$ and the velocity caused by an arbitrary helix rotation $\omega$ :

$$
\mathbf{v}_{s}=\mathbf{v}+\boldsymbol{\omega} \times \mathbf{p}(\phi)=\mathbf{v}-\mathbf{p}(\phi) \times \omega .
$$

This can be expressed in the helix frame as:

$$
{ }^{\mathrm{h}} \mathbf{v}_{s}={ }^{\mathrm{h}} \mathbf{v}-\mathbb{S}\left\{{ }^{\mathrm{h}} \mathbf{p}(\phi)\right\}^{\mathrm{h}} \boldsymbol{\omega}={ }^{\mathrm{h}} \mathbf{v}+\mathbb{S}\left\{{ }^{\mathrm{h}} \mathbf{p}(\phi)\right\}^{\mathrm{Th}} \boldsymbol{\omega},
$$

where $\mathbb{S}\{\mathbf{k}\}$ packs the vector $\mathbf{k}=\left[\begin{array}{lll}k_{1} & k_{2} & k_{3}\end{array}\right]^{\mathrm{T}}$ into the skew-symmetric matrix, representing the cross-product operation:

$$
\mathbb{S}\{\mathbf{k}\}=\left[\begin{array}{ccc}
0 & -k_{3} & k_{2} \\
k_{3} & 0 & -k_{1} \\
-k_{2} & k_{1} & 0
\end{array}\right] .
$$

Substituting (10) into (7) results in:

$$
{ }^{\mathrm{h}} \mathrm{d} \mathbf{f}_{s}={ }^{\mathrm{h}} \boldsymbol{\Xi}(\phi)^{\mathrm{h}} \mathbf{v} \mathrm{d} s+{ }^{\mathrm{h}} \boldsymbol{\Xi}(\phi) \mathbb{S}\left\{{ }^{\mathrm{h}} \mathbf{p}(\phi)\right\}^{\mathrm{Th}} \boldsymbol{\omega} \mathrm{d} s .
$$

A similar differential expression relating fluidic torque to translational and angular velocities of the helix can also be derived. Each force acting on an infinitesimally small segment of helix induces a subsequent torque about the helix origin. The relationship between the torque and force at an arbitrary helix segment, parameterized by $\phi$, can be expressed in the helix frame as:

$$
{ }^{\mathrm{h}} \mathrm{d} \boldsymbol{\tau}_{s}={ }^{\mathrm{h}} \mathbf{p}(\phi) \times{ }^{\mathrm{h}} \mathrm{d} \mathbf{f}_{s}=\mathbb{S}\left\{{ }^{\mathrm{h}} \mathbf{p}(\phi)\right\}^{\mathrm{h}} \mathrm{d} \mathbf{f}_{s} .
$$

Here, we make an assumption that is common when using RFT: that the drag torque on the helix due to a given segment is due to the translational velocity of the segment as it slices through the fluid and that the torque due to the small segment's rotation is negligible. This assumption significantly simplifies calculations and should be valid provided that the cross-section of the segment is small compared to the overall radius of the helix.

The total fluidic force and torque on the helix are found by integrating the forces and torques on the segments along the total length of the helix:

$$
\mathbf{f}_{\mathrm{h}}=\int \mathrm{d} \mathbf{f}_{s}, \quad \boldsymbol{\tau}_{\mathrm{h}}=\int \mathrm{d} \boldsymbol{\tau}_{s} .
$$

For both (12) and (13) the helix was parameterized using the polar angle $\phi$. Therefore, the force and torque will be obtained by integrating both differentials with respect to $\phi$. Substituting (12) into (13), replacing $\mathrm{d} s$ with $r_{\mathrm{h}} \mathrm{d} \phi / \sin (\theta)$ and integrating $\phi$ from $-\pi n$ to $\pi n$ produces two integral equations for an $n$-turn helix 
centered at $\mathbf{O}_{\mathrm{h}}$ :

$$
\begin{gathered}
\mathrm{h}_{\mathbf{f}_{\mathrm{h}}=}\left(\frac{r_{\mathrm{h}}}{\sin (\theta)} \int_{-\pi n}^{\pi n}{ }^{\mathrm{h}} \boldsymbol{\Xi}(\phi) \mathrm{d} \phi\right){ }^{\mathrm{h}} \mathbf{v} \\
+\left(\frac{r_{\mathrm{h}}}{\sin (\theta)} \int_{-\pi n}^{\pi n}{ }^{\mathrm{h}} \boldsymbol{\Xi}(\phi) \mathbb{S}\left\{{ }^{\mathrm{h}} \mathbf{p}(\phi)\right\}^{\mathrm{T}} \mathrm{d} \phi\right){ }^{\mathrm{h}} \boldsymbol{\omega} \\
\mathrm{h}_{\boldsymbol{\tau}_{\mathrm{h}}=}\left(\frac{r_{\mathrm{h}}}{\sin (\theta)} \int_{-\pi n}^{\pi n} \mathbb{S}\left\{{ }^{\mathrm{h}} \mathbf{p}(\phi)\right\}^{\mathrm{h}} \boldsymbol{\Xi}(\phi) \mathrm{d} \phi\right){ }^{\mathrm{h}} \mathbf{v} \\
+\left(\frac{r_{\mathrm{h}}}{\sin (\theta)} \int_{-\pi n}^{\pi n} \mathbb{S}\left\{{ }^{\mathrm{h}} \mathbf{p}(\phi)\right\}^{\mathrm{h}} \boldsymbol{\Xi}(\phi) \mathbb{S}\left\{{ }^{\mathrm{h}} \mathbf{p}(\phi)\right\}^{\mathrm{T}} \mathrm{d} \phi\right){ }^{\mathrm{h}} \boldsymbol{\omega} .
\end{gathered}
$$

Evaluating all four matrix integrals results in two equations relating force and torque to translational velocity and angular velocity around $\mathbf{O}_{\mathrm{h}}$ :

$$
\left[\begin{array}{c}
{ }^{{ }^{h}} \mathbf{f}_{\mathrm{h}} \\
{ }^{{ }^{h}} \boldsymbol{\tau}_{\mathrm{h}}
\end{array}\right]=\left[\begin{array}{ll}
{ }^{\mathrm{h}} \mathbf{A}_{\mathrm{h}} & { }^{\mathrm{h}} \mathbf{B}_{\mathrm{h}} \\
{ }^{\mathrm{h}} \mathbf{B}_{\mathrm{h}}^{\mathrm{T}} & { }^{\mathrm{h}} \mathbf{C}_{\mathrm{h}}
\end{array}\right]\left[\begin{array}{c}
{ }^{\mathrm{h}} \mathbf{v} \\
{ }^{\mathrm{h}} \boldsymbol{\omega}
\end{array}\right],
$$

where:

$$
\begin{array}{rlr}
{ }^{\mathrm{h}} \mathbf{A}_{\mathrm{h}} & =\left[\begin{array}{ccc}
a_{\mathrm{h} 11} & 0 & 0 \\
0 & a_{\mathrm{h} 22} & 0 \\
0 & 0 & a_{\mathrm{h} 22}
\end{array}\right], \\
{ }^{\mathrm{h}} \mathbf{C}_{\mathrm{h}} & =\left[\begin{array}{ccc}
c_{\mathrm{h} 11} & 0 & c_{\mathrm{h} 13} \\
0 & c_{\mathrm{h} 22} & 0 \\
c_{\mathrm{h} 13} & 0 & c_{\mathrm{h} 33}
\end{array}\right], & { }^{\mathrm{h}} \mathbf{B}_{\mathrm{h}}=\left[\begin{array}{ccc}
b_{\mathrm{h} 11} & 0 & b_{\mathrm{h} 13} \\
0 & b_{\mathrm{h} 22} & 0 \\
0 & 0 & b_{\mathrm{h} 33}
\end{array}\right]
\end{array}
$$

and:

$$
\begin{array}{r}
a_{\mathrm{h} 11}=\frac{2 \pi n r_{\mathrm{h}}\left(\xi_{\|} \cos ^{2}(\theta)+\xi_{\perp} \sin ^{2}(\theta)\right)}{\sin (\theta)} \\
a_{\mathrm{h} 22}=\frac{\pi n r_{\mathrm{h}}\left(\xi_{\perp}+\xi_{\perp} \cos ^{2}(\theta)+\xi_{\|} \sin ^{2}(\theta)\right)}{\sin (\theta)} \\
b_{\mathrm{h} 11}=2 \pi n r_{\mathrm{h}}^{2}\left(\xi_{\|}-\xi_{\perp}\right) \cos (\theta) \\
b_{\mathrm{h} 13}=\frac{-2 \pi n r_{\mathrm{h}}^{2}\left(\xi_{\|}-\xi_{\perp}\right) \cos (\theta)}{\tan (\theta)} \\
b_{\mathrm{h} 22}=\frac{-3 \pi n r_{\mathrm{h}}^{2}\left(\xi_{\|}-\xi_{\perp}\right) \cos (\theta)}{2} \\
b_{\mathrm{h} 33}=\frac{-\pi n r_{\mathrm{h}}^{2}\left(\xi_{\|}-\xi_{\perp}\right) \cos (\theta)}{2} \\
c_{\mathrm{h} 11}=\frac{2 \pi n r_{\mathrm{h}}^{3}\left(\xi_{\perp} \cos ^{2}(\theta)+\xi_{\|} \sin ^{2}(\theta)\right)}{\sin (\theta)} \\
c_{\mathrm{h} 13}=\frac{-2 \pi n r_{\mathrm{h}}^{3}\left(\xi_{\perp} \cos ^{2}(\theta)+\xi_{\|} \sin ^{2}(\theta)\right)}{\sin (\theta) \tan (\theta)}
\end{array}
$$




$$
\begin{aligned}
c_{\mathrm{h} 22}= & \frac{2 \pi n r_{\mathrm{h}}^{3}\left(\xi_{\|} \cos ^{2}(\theta)+\xi_{\perp} \sin ^{2}(\theta)-\xi_{\perp} / 2\right)}{\sin (\theta)} \\
& +\frac{\pi n r_{\mathrm{h}}^{3}\left(\xi_{\|} \cos ^{2}(\theta)-\xi_{\perp} \sin ^{2}(\theta)-\xi_{\perp}\right)}{2 \tan ^{2}(\theta) \sin (\theta)} \\
& +\frac{\left(\pi n r_{\mathrm{h}}\right)^{3}\left(\xi_{\|} \cos ^{2}(\theta)-\xi_{\perp} \sin ^{2}(\theta)+\xi_{\perp}\right)}{3 \tan ^{2}(\theta) \sin (\theta)} \\
c_{\mathrm{h} 33}= & \frac{\pi n r_{\mathrm{h}}^{3} \xi_{\perp}}{\sin (\theta)}-\frac{\pi n r_{\mathrm{h}}^{3}\left(\xi_{\perp} \cos ^{2}(\theta)+\xi_{\|} \sin ^{2}(\theta)-\xi_{\perp}\right)}{2 \tan ^{2}(\theta) \sin (\theta)} \\
+ & \frac{\left(\pi n r_{\mathrm{h}}\right)^{3}\left(\xi_{\perp} \cos ^{2}(\theta)+\xi_{\|} \sin ^{2}(\theta)+\xi_{\perp}\right)}{3 \tan ^{2}(\theta) \sin (\theta)} .
\end{aligned}
$$

As the microswimmer rotates about $\mathbf{O}_{\mathrm{h}}$, the spherical magnetic head of radius $r_{\mathrm{m}}$ fixed to the helix exerts fluidic forces and torques on the microswimmer that we assume to be independent from those caused by the helix. Let $\mathbf{k}$ be the vector from the center of the helix $\mathbf{O}_{\mathrm{h}}$ to the center of the magnetic head $\mathbf{O}_{\mathrm{m}}$ as shown in Fig. 2. Given that the translational and rotational drag coefficients in Stokes flow of viscosity $\eta$ are $\xi_{v \mathrm{~m}}=6 \pi \eta r_{\mathrm{m}}$ and $\xi_{\omega \mathrm{m}}=8 \pi \eta r_{m}^{3}$, respectively [17], the drag force acting on the head is the product of the translational drag coefficient and the head's velocity. Arbitrary movement of the microswimmer produces a magnet velocity, expressed in the helix frame, of

$$
{ }^{\mathrm{h}} \mathbf{v}_{\mathrm{m}}={ }^{\mathrm{h}} \mathbf{v}+{ }^{\mathrm{h}} \boldsymbol{\omega} \times{ }^{\mathrm{h}} \mathbf{k}={ }^{\mathrm{h}} \mathbf{v}-{ }^{\mathrm{h}} \mathbf{k} \times{ }^{\mathrm{h}} \boldsymbol{\omega}={ }^{\mathrm{h}} \mathbf{v}+\mathbb{S}\left\{{ }^{\mathrm{h}} \mathbf{k}\right\}{ }^{\mathrm{Th}} \boldsymbol{\omega},
$$

which corresponds to a force on the magnet:

$$
{ }^{\mathrm{h}} \mathbf{f}_{\mathrm{m}}=\xi_{v \mathrm{~m}}{ }^{\mathrm{h}} \mathbf{v}+\xi_{v \mathrm{~m}} \mathbb{S}\left\{{ }^{\mathrm{h}} \mathbf{k}\right\}^{\mathrm{Th}} \boldsymbol{\omega} .
$$

The drag torque caused by the spherical head is a result of the force acting at the moment arm $\mathbf{k}$ as well as the drag caused by the rotation of the head itself. This can be expressed in the form ${ }^{\mathrm{h}} \boldsymbol{\tau}_{\mathrm{m}}={ }^{\mathrm{h}} \mathbf{k} \times{ }^{\mathrm{h}} \mathbf{f}_{\mathrm{m}}+\xi_{\omega \mathrm{m}}{ }^{\mathrm{h}} \boldsymbol{\omega}$, with ${ }^{\mathrm{h}} \mathbf{f}_{\mathrm{m}}$ from (32). Replacing the cross-product operator with its corresponding skew-symmetric matrix and grouping like terms produces:

$$
{ }^{\mathrm{h}} \boldsymbol{\tau}_{\mathrm{m}}=\xi_{v \mathrm{~m}} \mathbb{S}\left\{{ }^{\mathrm{h}} \mathbf{k}\right\}^{\mathrm{h}} \mathbf{v}+\left(\xi_{v \mathrm{~m}} \mathbb{S}\left\{{ }^{\mathrm{h}} \mathbf{k}\right\} \mathbb{S}\left\{{ }^{\mathrm{h}} \mathbf{k}\right\}^{\mathrm{T}}+\xi_{\omega \mathrm{m}} \mathbf{I}\right)^{\mathrm{h}} \boldsymbol{\omega}
$$

where I is the identity matrix. If we let the matrices:

$$
\begin{aligned}
& { }^{\mathrm{h}} \mathbf{A}_{\mathrm{m}}=\xi_{v \mathrm{~m}} \mathbf{I}, \quad{ }^{\mathrm{h}} \mathbf{B}_{\mathrm{m}}=\xi_{v \mathrm{~m}} \mathbb{S}\left\{{ }^{\mathrm{h}} \mathbf{k}\right\}^{\mathrm{T}} \\
& { }^{\mathrm{h}} \mathbf{C}_{\mathrm{m}}=\xi_{v \mathrm{~m}} \mathbb{S}\left\{{ }^{\mathrm{h}} \mathbf{k}\right\} \mathbb{S}\left\{{ }^{\mathrm{h}} \mathbf{k}\right\}^{\mathrm{T}}+\xi_{\omega \mathrm{m}} \mathbf{I},
\end{aligned}
$$

then the total force ${ }^{\mathrm{h}} \mathbf{f}={ }^{\mathrm{h}} \mathbf{f}_{\mathrm{h}}+{ }^{\mathrm{h}} \mathbf{f}_{\mathrm{m}}$ and torque ${ }^{\mathrm{h}} \boldsymbol{\tau}={ }^{\mathrm{h}} \boldsymbol{\tau}_{\mathrm{h}}+{ }^{\mathrm{h}} \boldsymbol{\tau}_{\mathrm{m}}$ acting on the microswimmer are:

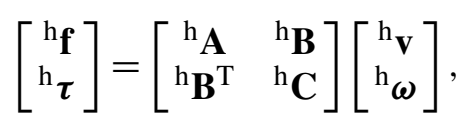


where ${ }^{\mathrm{h}} \mathbf{A}={ }^{\mathrm{h}} \mathbf{A}_{\mathrm{h}}+{ }^{\mathrm{h}} \mathbf{A}_{\mathrm{m}},{ }^{\mathrm{h}} \mathbf{B}={ }^{\mathrm{h}} \mathbf{B}_{\mathrm{h}}+{ }^{\mathrm{h}} \mathbf{B}_{\mathrm{m}}$ and ${ }^{\mathrm{h}} \mathbf{C}={ }^{\mathrm{h}} \mathbf{C}_{\mathrm{h}}+{ }^{\mathrm{h}} \mathbf{C}_{\mathrm{m}}$ :

$$
\begin{aligned}
& \mathrm{h}_{\mathbf{A}}= {\left[\begin{array}{ccc}
a_{11} & 0 & 0 \\
0 & a_{22} & 0 \\
0 & 0 & a_{22}
\end{array}\right]=\left[\begin{array}{ccc}
a_{\mathrm{h} 11}+\xi_{v \mathrm{~m}} & 0 & 0 \\
0 & a_{\mathrm{h} 22}+\xi_{v \mathrm{~m}} & 0 \\
0 & 0 & a_{\mathrm{h} 22}+\xi_{v \mathrm{~m}}
\end{array}\right] } \\
&{ }^{\mathrm{h}} \mathbf{B}=\left[\begin{array}{ccc}
b_{11} & 0 & b_{13} \\
0 & b_{22} & b_{23} \\
0 & -b_{23} & b_{33}
\end{array}\right]=\left[\begin{array}{ccc}
b_{\mathrm{h} 11} & 0 & b_{\mathrm{h} 13} \\
0 & b_{\mathrm{h} 22} & \xi_{v \mathrm{~m}}|\mathbf{k}| \\
0 & -\xi_{v \mathrm{~m}}|\mathbf{k}| & b_{\mathrm{h} 33}
\end{array}\right] \\
& \mathrm{h}_{\mathbf{C}}=\left[\begin{array}{ccc}
c_{11} & 0 & c_{13} \\
0 & c_{22} & 0 \\
c_{13} & 0 & c_{33}
\end{array}\right] \\
&=\left[\begin{array}{ccc}
c_{\mathrm{h} 11}+\xi_{\omega \mathrm{m}} & 0 \\
0 & c_{\mathrm{h} 22}+\xi_{v \mathrm{~m}}|\mathbf{k}|^{2}+\xi_{\omega \mathrm{m}} & c_{\mathrm{h} 13} \\
c_{\mathrm{h} 13} & 0
\end{array}\right] .
\end{aligned}
$$

Equation (35) is the 6 d.o.f. equivalent of (1). Note that $\mathbf{f}$ and $\boldsymbol{\tau}$ do not represent viscous drag associated with the microswimmer's total velocity $\mathbf{v}$ and $\boldsymbol{\omega}$. Rather, they represent non-fluidic force and torque - in this paper, force due to gravity and torque due to magnetism - corresponding to a given microswimmer velocity (i.e., the force and torque required to overcome viscous drag for a given microswimmer velocity). Now that we are equipped with a 6-d.o.f. helical microswimmer model, we are ready to develop the control algorithm that enables intuitive control over the microswimmer's velocity, as opposed to directly controlling the microswimmer's orientation and rotation speed.

\section{Algorithm for Velocity Control with Gravity Compensation}

To compensate for the drift caused by an applied force on the microswimmer, it is necessary to find the direction that it must point, $\mathbf{x}_{\mathrm{h}}$, and at what speed it must rotate, $\Omega$, to achieve a desired spatial velocity, ${ }^{\mathrm{w}} \mathbf{v}$, where $\mathrm{w}$ indicates the stationary world frame. In this paper, the microswimmer's weight is the sole applied non-fluidic force, represented by a vector $m \mathbf{g}$, where $m$ is the mass of the microswimmer and $\mathbf{g}$ is the acceleration of gravity, and the 'downward' direction is defined with the unit vector $\hat{\mathbf{g}}=\mathbf{g} /\|\mathbf{g}\|$. As can be seen from the off-diagonal terms in the ${ }^{\mathrm{h}} \mathbf{B}$ and ${ }^{\mathrm{h}} \mathbf{C}$ matrices of Section 2, the result of steering the microswimmer (i.e., rotating the swimmer around the $\mathbf{y}_{\mathrm{h}}$ or $\mathbf{z}_{\mathrm{h}}$ axis) can produce complex forces and torques acting on the microswimmer aside from those produced by weight. Despite this fact, previous research has shown that magnetic helical microswimmers can be turned effectively by adjusting the axis around which the applied magnetic field rotates $[4,12]$. Although there is evidence that commanding steering maneuvers that are too rapid can lead to loss of control, we make the assumption herein that the microswimmer can be turned continuously to a desired pitch angle away from vertical fast enough such that the microswimmer's transient behavior is negligible.

In this section, we present a steady-state solution to the velocity problem, and use it to produce a control algorithm that takes a desired velocity as its input and outputs 
the direction the microswimmer must be pointed and how fast it must be rotating to attain the desired velocity at steady state. We denote the pointing direction by $\tilde{\mathbf{x}}$ and the rotation speed by $\Omega$. In the context of command inputs using magnetic fields, $\tilde{\mathbf{x}}$ is also the axis around which the magnetic field should rotate, such that the magnetic field is always perpendicular to $\tilde{\mathbf{x}}$ and $\Omega$ is the rate of rotation.

If the stationary world frame is aligned with the microswimmer's workspace in an intuitive manner, the control inputs would best be expressed in this frame. To avoid requiring vectors to be converted back and forth between the world frame and the microswimmer's coordinate frame (i.e., frame h, which rotates), any equations used to generate the necessary pitch and angular velocity would also best be represented in the world frame. From the 6-d.o.f. model derived in Section 2, the portion of (35) that is of particular interested to us is:

$$
{ }^{\mathrm{h}} \mathbf{f}={ }^{\mathrm{h}} \mathbf{A}^{\mathrm{h}} \mathbf{v}+{ }^{\mathrm{h}} \mathbf{B}^{\mathrm{h}} \boldsymbol{\omega},
$$

which relates the microswimmer's velocity to applied non-fluidic forces and angular velocity of the microswimmer. As ${ }^{\mathrm{h}} \mathbf{A}$ is clearly invertible, the desired velocity can be solved for in (39) to produce:

$$
{ }^{\mathrm{h}} \mathbf{v}=\left({ }^{\mathrm{h}} \mathbf{A}^{-1}\right){ }^{\mathrm{h}} \mathbf{f}+\left(-{ }^{\mathrm{h}} \mathbf{A}^{-1 \mathrm{~h}} \mathbf{B}\right)^{\mathrm{h}} \boldsymbol{\omega}={ }^{\mathrm{h}} \mathbf{D}^{\mathrm{h}} \mathbf{f}+{ }^{\mathrm{h}} \mathbf{E}^{\mathrm{h}} \boldsymbol{\omega},
$$

where:

$$
{ }^{\mathrm{h}} \mathbf{D}=\left[\begin{array}{ccc}
d_{11} & 0 & 0 \\
0 & d_{22} & 0 \\
0 & 0 & d_{22}
\end{array}\right], \quad{ }^{\mathrm{h}} \mathbf{E}=\left[\begin{array}{ccc}
e_{11} & 0 & e_{13} \\
0 & e_{22} & e_{23} \\
0 & -e_{23} & e_{33}
\end{array}\right],
$$

all expressed in the microswimmer's helix frame. Using ${ }^{\mathrm{w}} \mathbf{R}_{\mathrm{h}}$, (40) can be written in the world frame:

$$
{ }^{\mathrm{w}} \mathbf{v}={ }^{\mathrm{w}} \mathbf{D}^{\mathrm{w}} \mathbf{f}+{ }^{\mathrm{w}} \mathbf{E}^{\mathrm{w}} \boldsymbol{\omega},
$$

using similarity transformations:

$$
\begin{aligned}
& { }^{\mathrm{w}} \mathbf{v}={ }^{\mathrm{w}} \mathbf{R}_{\mathrm{h}}{ }^{\mathrm{h}} \mathbf{v}, \quad{ }^{\mathrm{w}} \mathbf{f}={ }^{\mathrm{w}} \mathbf{R}_{\mathrm{h}}{ }^{\mathrm{h}} \mathbf{f} \\
& { }^{\mathrm{w}} \mathbf{D}={ }^{\mathrm{w}} \mathbf{R}_{\mathrm{h}}{ }^{\mathrm{h}} \mathbf{D}^{\mathrm{h}} \mathbf{R}_{\mathrm{w}}, \quad{ }^{\mathrm{w}} \mathbf{E}={ }^{\mathrm{w}} \mathbf{R}_{\mathrm{h}}{ }^{\mathrm{h}} \mathbf{E}^{\mathrm{h}} \mathbf{R}_{\mathrm{w}} .
\end{aligned}
$$

Constructing ${ }^{\mathrm{h}} \mathbf{R}_{\mathrm{w}}$ requires the orientation of the robot as it rotates to be detected. In practice, it is difficult to sense the orientation of the robot about its central axis as it rotates during propulsion (e.g., using computer vision). To make best use of (42) for anything aside from the trivial cases where the microswimmer is being commanded to move vertically, we will express (42) in a manner that does not require the orientation of the microswimmer about its central axis to be known.

Since weight is the sole non-fluidic force acting upon the microrobot, the input force $\mathbf{f}$ to (39) is $m \mathbf{g}$. The desired velocity, $\mathbf{v}$, can be broken into vertical and horizontal components, respectively:

$$
\begin{gathered}
\mathbf{v}_{\mathrm{ver}}=(\mathbf{v} \cdot \hat{\mathbf{g}}) \hat{\mathbf{g}} \\
\mathbf{v}_{\text {hor }}=\mathbf{v}-\mathbf{v}_{\text {ver }} .
\end{gathered}
$$


If $\left\|\mathbf{v}_{\text {hor }}\right\|=0$, then the microswimmer is being commanded to move vertically: either straight up, straight down or a stationary hover. This is the trivial case where (39) effectively becomes equivalent to its 2-d.o.f. counterpart from (1), making the solution for $\tilde{\mathbf{x}}$ and $\Omega$ straightforward:

$$
\Omega=\frac{\|\mathbf{v}\|+d_{11}\|\mathbf{f}\|}{e_{11}} .
$$

If $\left\|\mathbf{v}_{\text {hor }}\right\| \neq 0$, the solution is more complicated. Using $\hat{\mathbf{g}}$, we will construct a new coordinate frame associated with the microswimmer that does not rotate when the microswimmer rotates about its central axis and we will express (42) in this frame. This coordinate system (denoted by $p$ ) will be constructed such that the basis vectors are eigenvectors of ${ }^{\mathrm{w}} \mathbf{D}$ or ${ }^{\mathrm{w}} \mathbf{E}$. As the eigenvectors of ${ }^{\mathrm{w}} \mathbf{D}$ or ${ }^{\mathrm{w}} \mathbf{E}$ are aligned with the principle directions of the microswimmer, we will refer to this frame as the 'principle' coordinate frame. Let the basis of this coordinate frame be defined as:

$$
\begin{aligned}
\mathbf{x}_{\mathrm{p}} & =\frac{\left(\mathbf{x}_{\mathrm{h}} \cdot \mathbf{v}\right) \mathbf{x}_{\mathrm{h}}}{\left|\mathbf{x}_{\mathrm{h}} \cdot \mathbf{v}\right|} \\
\mathbf{y}_{\mathrm{p}} & =\frac{\mathbf{x}_{\mathrm{p}} \times \mathbf{g}}{\left\|\mathbf{x}_{\mathrm{p}} \times \mathbf{g}\right\|} \\
\mathbf{z}_{\mathrm{p}} & =\mathbf{x}_{\mathrm{p}} \times \mathbf{y}_{\mathrm{p}} .
\end{aligned}
$$

The principle frame is depicted in Fig. 3a. Expressing (42) in the principle coordinate frame transforms the problem of finding the necessary pitch and angular velocity into one that is relatively easy to solve and, as will be seen, is invariant to rotations of the microswimmer about its central axis. The representation of (42) in terms of the principle frame can be found by determining the representation of its terms, ${ }^{\mathrm{w}} \mathbf{D}^{\mathrm{w}} \mathbf{f}$ and ${ }^{\mathrm{w}} \mathbf{E}^{\mathrm{w}} \boldsymbol{\omega}$, individually.

If we make the assumption that the swimmer is at steady state as discussed earlier, then we know ${ }^{\mathrm{w}} \boldsymbol{\omega}=\Omega^{\mathrm{w}} \tilde{\mathbf{x}}=\Omega^{\mathrm{w}} \mathbf{x}_{\mathrm{p}}$ and ${ }^{\mathrm{w}} \mathbf{x}_{\mathrm{p}}$ is parallel to ${ }^{\mathrm{w}} \mathbf{x}_{\mathrm{h}}$ (i.e., ${ }^{\mathrm{w}} \mathbf{x}_{\mathrm{p}}={ }^{\mathrm{w}} \mathbf{x}_{\mathrm{h}}$ or

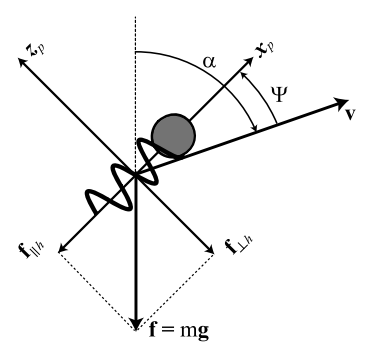

(a)

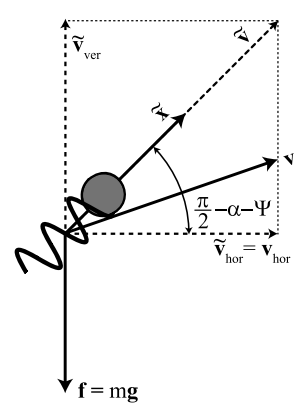

(b)

Figure 3. (a) Definition of the principle frame, angles and forces. (b) Construction of $\tilde{\mathbf{x}}$. 
${ }^{\mathrm{w}} \mathbf{x}_{\mathrm{p}}=-{ }^{\mathrm{w}} \mathbf{x}_{\mathrm{h}}$ ). It can be easily verified that ${ }^{\mathrm{h}} \mathbf{x}_{\mathrm{h}}$ is an eigenvector of ${ }^{\mathrm{h}} \mathbf{E}$ corresponding to the eigenvalue $e_{11}$. The similarity transformations of (43) do not affect the eigenvalues of matrices and only rotate the eigenvectors with the ${ }^{\mathrm{w}} \mathbf{R}_{\mathrm{h}}$ transformation, implying that $e_{11}$ is an eigenvalue of ${ }^{\mathrm{w}} \mathbf{E}$ and its corresponding eigenvector is ${ }^{\mathrm{w}} \mathbf{x}_{\mathrm{h}}$, and due to their parallelism, ${ }^{\mathrm{w}} \mathbf{x}_{\mathrm{p}}$ is also a corresponding eigenvector. Thus, the expression of ${ }^{\mathrm{w}} \mathbf{E}^{\mathrm{w}} \boldsymbol{\omega}$ using the principle frame basis vectors can be easily found:

$$
{ }^{\mathrm{w}} \mathbf{E}^{\mathrm{w}} \boldsymbol{\omega}={ }^{\mathrm{w}} \mathbf{E} \Omega^{\mathrm{w}} \mathbf{x}_{\mathrm{p}}=e_{11} \Omega^{\mathrm{w}} \mathbf{x}_{\mathrm{p}} .
$$

Recasting the ${ }^{\mathrm{w}} \mathbf{D}^{\mathrm{w}} \mathbf{f}$ term of (42) is performed in a similar manner. It is also easily verified that ${ }^{\mathrm{h}} \mathbf{D}$ has two eigenvalues: $d_{11}$ and $d_{22}$. The $d_{11}$ eigenvalue has the corresponding eigenvector ${ }^{h} \mathbf{x}_{h}$ and an eigenspace spanned by $\left\{{ }^{\mathrm{h}} \mathbf{y}_{\mathrm{h}},{ }^{\mathrm{h}} \mathbf{z}_{\mathrm{h}}\right\}$ corresponds to the eigenvalue $d_{22}$. Any vector that is a linear combination of ${ }^{\mathrm{h}} \mathbf{y}_{\mathrm{h}}$ and ${ }^{\mathrm{h}} \mathbf{z}_{\mathrm{h}}$ will be scaled by the eigenvalue $d_{22}$. Again, eigenvalues are preserved under similarity transformations, so the subsequent eigenvalues and eigenvectors of ${ }^{\mathrm{w}} \mathbf{D}$ are $d_{11}$ corresponding to ${ }^{\mathrm{w}} \mathbf{x}_{\mathrm{h}}$ and $d_{22}$ corresponding to any vector found in the span of $\left\{{ }^{\mathrm{w}} \mathbf{y}_{\mathrm{h}},{ }^{\mathrm{w}} \mathbf{z}_{\mathrm{h}}\right\}$. The force ${ }^{\mathrm{w}} \mathbf{f}$ acting on the microswimmer can be broken into two component vectors with one parallel to the eigenvector ${ }^{\mathrm{w}} \mathbf{x}_{\mathrm{h}}$, which is aligned with the central axis of the helix, and the other perpendicular (i.e., in the span of ${ }^{\mathrm{s}} \mathbf{y}_{\mathrm{h}}$ and $\left.{ }^{\mathrm{s}} \mathbf{Z}_{\mathrm{h}}\right)$ :

$$
{ }^{\mathrm{w}} \mathbf{f}=\left(\left(\mathbf{f} \cdot \mathbf{x}_{\mathrm{h}}\right)^{\mathrm{w}} \mathbf{x}_{\mathrm{h}}\right)+\left(\left(\mathbf{f} \cdot \mathbf{y}_{\mathrm{h}}\right)^{\mathrm{w}} \mathbf{y}_{\mathrm{h}}+\left(\mathbf{f} \cdot \mathbf{z}_{\mathrm{h}}\right)^{\mathrm{w}} \mathbf{z}_{\mathrm{h}}\right)={ }^{\mathrm{w}} \mathbf{f}_{\| \mathrm{h}}+{ }^{\mathrm{s}} \mathbf{f}_{\perp \mathrm{h}} .
$$

Both ${ }^{\mathrm{w}} \mathbf{f}$ and ${ }^{\mathrm{w}} \mathbf{f}_{\| \mathrm{h}}$ are unaffected by the spinning of the microswimmer about the ${ }^{\mathrm{w}} \mathbf{x}_{\mathrm{h}}$ axis, implying that ${ }^{\mathrm{w}} \mathbf{f}_{\perp \mathrm{h}}$ remains unchanged as well. Using this fact, and because both ${ }^{\mathrm{w}} \mathbf{y}_{\mathrm{p}}$ and ${ }^{\mathrm{w}} \mathbf{z}_{\mathrm{p}}$ are in the span of $\left\{{ }^{\mathrm{w}} \mathbf{y}_{\mathrm{h}},{ }^{\mathrm{w}} \mathbf{z}_{\mathrm{h}}\right\},{ }^{\mathrm{w}} \mathbf{f}_{\perp \mathrm{h}}$ can be written as linear combinations of ${ }^{\mathrm{w}} \mathbf{y}_{\mathrm{p}}$ and ${ }^{\mathrm{w}} \mathbf{z}_{\mathrm{p}}$. In fact, as can be seen from Fig. 3, no component of ${ }^{\mathrm{w}} \mathbf{f}_{\perp \mathrm{h}}$ lies in the direction of ${ }^{\mathrm{w}} \mathbf{y}_{\mathrm{p}}$, making ${ }^{\mathrm{w}} \mathbf{f}_{\perp \mathrm{h}}=\left(\mathbf{f} \cdot \mathbf{z}_{\mathrm{p}}\right)^{\mathrm{w}} \mathbf{z}_{\mathrm{p}}$. This implies that ${ }^{\mathrm{w}} \mathbf{f}_{\perp \mathrm{h}}$ is an eigenvector corresponding to eigenvalue $d_{22}$ and, along with the fact that ${ }^{\mathrm{w}} \mathbf{f}_{\| \mathrm{h}}$ is an eigenvector corresponding to eigenvalue $d_{11}$, leads us to write the ${ }^{\mathrm{w}} \mathbf{D}^{\mathrm{w}} \mathbf{f}$ term of (42) using the principle frame basis:

$$
\begin{aligned}
{ }^{\mathrm{w}} \mathbf{D}^{\mathrm{w}} \mathbf{f} & ={ }^{\mathrm{w}} \mathbf{D}^{\mathrm{w}} \mathbf{f}_{\| \mathrm{h}}+{ }^{\mathrm{w}} \mathbf{D}^{\mathrm{w}} \mathbf{f}_{\perp \mathrm{h}}=d_{11}{ }^{\mathrm{w}} \mathbf{f}_{\| \mathrm{h}}+d_{22}{ }^{\mathrm{w}} \mathbf{f}_{\perp \mathrm{h}} \\
& =d_{11}\left(\mathbf{f} \cdot \mathbf{x}_{\mathrm{p}}\right){ }^{\mathrm{w}} \mathbf{x}_{\mathrm{p}}+d_{22}\left(\mathbf{f} \cdot \mathbf{z}_{\mathrm{p}}\right){ }^{\mathrm{w}} \mathbf{z}_{\mathrm{p}} .
\end{aligned}
$$

Combining (51) and (53) produces (42) written using the basis vectors of the principle frame:

$$
{ }^{\mathrm{w}} \mathbf{v}=d_{11}\left(\mathbf{f} \cdot \mathbf{x}_{\mathrm{p}}\right)^{\mathrm{w}} \mathbf{x}_{\mathrm{p}}+d_{22}\left(\mathbf{f} \cdot \mathbf{z}_{\mathrm{p}}\right)^{\mathrm{w}} \mathbf{z}_{\mathrm{p}}+e_{11} \Omega^{\mathrm{w}} \mathbf{x}_{\mathrm{p}}
$$

As $\left(\mathbf{f} \cdot \mathbf{z}_{\mathrm{p}}\right)^{\mathrm{w}} \mathbf{z}_{\mathrm{p}}={ }^{\mathrm{w}} \mathbf{f}_{\perp \mathrm{h}}$ is invariant to the microswimmer's propulsive rotation and the swimmer rotates around the ${ }^{\mathrm{w}} \mathbf{x}_{\mathrm{p}}$ axis, neither of the terms in (54) are affected by the rotation of the swimmer about its central axis.

Since the horizontal component of the desired velocity $\mathbf{v}_{\text {hor }}$ is nonzero, we can denote the angle of $\mathbf{v}$ measured from the vertical axis of the world frame by $\alpha=$ $\tan ^{-1}\left(\left\|\mathbf{v}_{\text {hor }}\right\| /\left\|\mathbf{v}_{\text {ver }}\right\|\right)$. To compensate for weight, the microswimmer will need to be pitched upward by some angle above $\mathbf{v}$ as shown in Fig. 3. Let this angle be 
denoted by $\Psi$. Taking (54) and projecting it first onto the ${ }^{\mathrm{w}} \mathbf{x}_{\mathrm{p}}$ axis and then the ${ }^{\mathrm{w}} \mathbf{z}_{\mathrm{p}}$ axis produces two scalar equations of the form:

$$
\begin{gathered}
\left(\mathbf{v} \cdot \mathbf{x}_{\mathrm{p}}\right)=d_{11}\left(\mathbf{f} \cdot \mathbf{x}_{\mathrm{p}}\right)+e_{11} \Omega \\
\left(\mathbf{v} \cdot \mathbf{z}_{\mathrm{p}}\right)=d_{22}\left(\mathbf{f} \cdot \mathbf{z}_{\mathrm{p}}\right) .
\end{gathered}
$$

The angle $\Psi$ can be computed from (56) by recognizing from Fig. 2 that $\left(\mathbf{v} \cdot \mathbf{z}_{\mathrm{p}}\right)=$ $-\|\mathbf{v}\| \sin (\Psi)$ and $\left(\mathbf{f} \cdot \mathbf{z}_{\mathrm{p}}\right)=\|\mathbf{f}\| \sin (\Psi-\alpha)$, producing:

$$
-\|\mathbf{v}\| \sin (\Psi)=d_{22}\|\mathbf{f}\| \sin (\Psi-\alpha) \text {. }
$$

By utilizing the identity $\sin (\Psi-\alpha)=\sin (\Psi) \cos (\alpha)-\cos (\Psi) \sin (\alpha)$ and then grouping terms, we can solve for $\Psi$ resulting in:

$$
\Psi=\tan ^{-1}\left(\frac{d_{22}\|\mathbf{f}\| \sin (\alpha)}{\|\mathbf{v}\|+d_{22}\|\mathbf{f}\| \cos (\alpha)}\right)
$$

where the values for $\|\mathbf{v}\|,\|\mathbf{f}\|, d_{22}$ and $\alpha$ are all known. Using $\alpha$ and $\Psi$, the solution $\tilde{\mathbf{x}}$ can be reconstructed by defining an intermediate vector $\tilde{\mathbf{v}}=\tilde{\mathbf{v}}_{\text {hor }}+\tilde{\mathbf{v}}_{\text {ver }}$ composed of its horizontal and vertical components, respectively, where $\tilde{\mathbf{v}}_{\text {hor }}=\mathbf{v}_{\text {hor }}$ and $\tilde{\mathbf{v}}_{\text {ver }}=$ $-\left\|\tilde{\mathbf{v}}_{\text {hor }}\right\| \tan (\pi / 2-\alpha+\Psi) \hat{\mathbf{g}}$. The solution $\tilde{\mathbf{x}}$, then is computed as:

$$
\tilde{\mathbf{x}}=\frac{\tilde{\mathbf{v}}}{\|\tilde{\mathbf{v}}\|} \text {. }
$$

The construction of $\tilde{\mathbf{x}}$ is depicted in Fig. 3b. Once $\Psi$ has been determined, $\Omega$ can be found from (55) after recognizing that $\left(\mathbf{v} \cdot \mathbf{x}_{\mathrm{p}}\right)=\|\mathbf{v}\| \cos (\Psi)$ and $\left(\mathbf{f} \cdot \mathbf{x}_{\mathrm{p}}\right)=$ $-\|\mathbf{f}\| \cos (\Psi-\alpha)$ :

$$
\Omega=\frac{\|\mathbf{v}\| \cos (\Psi)+d_{11}\|\mathbf{f}\| \cos (\Psi-\alpha)}{e_{11}} .
$$

To summarize the control algorithm, the commanded velocity $\mathbf{v}$ is decomposed into vertical and horizontal components using (44) and (45), respectively. If $\left\|\mathbf{v}_{\text {hor }}\right\|=0$, then (46) and (47) are used to solve for $\tilde{\mathbf{x}}$ and $\Omega$, respectively. Otherwise, (59) and (60) are used. The magnetic field should then be perpendicular to and rotated about the axis $\tilde{\mathbf{x}}$ with a rotation speed of $\Omega \mathrm{rad} / \mathrm{s}$ to achieve the commanded velocity.

\section{Experimental Verification}

\subsection{Experimental Setup}

The experimental setup used to generate the controlled magnetic fields is shown in Fig. 4. The system consists of three nested sets of Helmholtz coils. Each set of Helmholtz coils generates a magnetic field that is optimally uniform in the center of the workspace, which is aligned with the axis of the coils and which varies linearly with the electrical current flowing through wire. Each set of Helmholtz coils is connected in series, such that a single current is used to power set. The three 


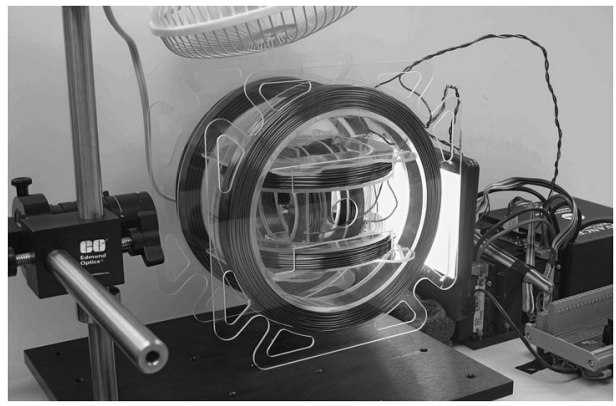

Figure 4. Experimental setup.

Table 1.

Parameters of Helmholtz coil electromagnets

\begin{tabular}{llllllc}
\hline $\begin{array}{l}\text { Coil } \\
\text { set }\end{array}$ & $\begin{array}{l}\text { Coil radius } \\
(\mathrm{mm})\end{array}$ & $\begin{array}{l}\text { No. wraps } \\
\text { (width) }\end{array}$ & $\begin{array}{l}\text { No. wraps } \\
\text { (depth) }\end{array}$ & $\begin{array}{l}\text { No. wraps } \\
\text { (total) }\end{array}$ & $\begin{array}{l}\text { Resistance } \\
(\Omega)\end{array}$ & $\begin{array}{l}\text { Inductance } \\
(\mathrm{mH})\end{array}$ \\
\hline Inner & 44 & 9 & 7 & 63 & 0.5 & 0.944 \\
Middle & 69 & 9 & 11 & 99 & 0.9 & 3.78 \\
Outer & 98 & 13 & 11 & 143 & 1.6 & 12.2 \\
\hline
\end{tabular}

The radius, number of wraps wide, number of wraps deep and total wraps are reported per individual coil, whereas the resistance and inductance are reported for each pair. All coils are wrapped with 14 AWG insulated copper magnet wire (1.628 mm diameter). The separation of the coils in a Helmholtz pair is equal to the radius of the coils in the pair.

sets are arranged orthogonally such that the magnetic field vector can be assigned arbitrarily, with each Helmholtz pair corresponding to one basis direction of the field vector. The details of the Helmholtz coils can be found in Table 1. Each set of Helmholtz coils is driven by an Advanced Motion Controls S16A8 PWM ana$\log$ servo drive, capable of $8 \mathrm{~A}$ continuous current and $16 \mathrm{~A}$ peak current. The S16A8 is designed to drive three-phase brushless motors with sinusoidal current profiles, which is similar to the task of generating rotating magnetic fields required herein. The three S16A8 drives are powered by a single Advanced Motion Controls PS2x300W power supply. Analog communication between the PC and the drives is accomplished with a Sensoray 626 Analog and Digital I/O card. The relationship between the generated magnetic field and the commanded current was calibrated in the center of the workspace using a Hirst GM08 gaussmeter. A $25 \mathrm{~mm} \times 25 \mathrm{~mm}$ square-cross-section vial with a length of $50 \mathrm{~mm}$ is located in the common center of the Helmholtz coils. A Basler A602FC camera fitted with a Computar MLH-10X macro zoom lens has a lateral view of the contents of the vial, which is backlit by a Coherent Cold Cathode ML-0405 Backlight Panel that gives high-contrast images. The entire system is cooled from above with a desk fan in a temperature-controlled room in order to maintain an approximately constant viscosity of the swimmer's 
environment. The system is controlled with a PC containing a 1.2-GHz dual-core processor and $2 \mathrm{~GB}$ of RAM running with Linux.

\subsection{Fabrication of the Magnetic Helical Swimmer}

The helical swimmer pictured in Fig. 5 was fabricated from Nitinol tubing with an outer diameter of $1.0 \mathrm{~mm}$ and inner diameter of $0.68 \mathrm{~mm}$ using micro wire electrical discharge machining (WEDM). The WEDM machine was designed and built at the University of Utah to utilize small-diameter wires ranging from 50 to $100 \mu \mathrm{m}$ [18]. For the helical cut shown in Fig. 5a, the machine was equipped with a rotary axis (A-axis) with its axis of rotation perpendicular to the EDM wire. In order to achieve a pitch of $1 \mathrm{~mm}$ and a helix ribbon width of $0.2 \mathrm{~mm}$, the $100-\mu \mathrm{m}$ wire first penetrated the tube to a depth of $0.15 \mathrm{~mm}$ below the axis of rotation ( $y$-axis). The helix was then cut in a single sweep by synchronizing the horizontal linear motion ( $x$-axis) with the rotation of the A-axis. Using a $100-\mu \mathrm{m}$ brass wire at $250 \mathrm{~V}$ open-circuit voltage and a 5.5-nF capacitor, the EDM cuts for the helical swimmer including a cylindrical cut for attaching the magnet took approximately $1 \mathrm{~h}$. The geometry of the helix was chosen to approximate the geometry of the microswimmers in Ref. [4].

As can been seen in Fig. 5a and b, a hemicylindrical pocket was machined into the 'head' of the helix. After fabricating the helix, a 1-mm long, 0.75-mm diameter axially magnetized cylindrical permanent magnet (nickel-plated NdFeB Grade N50 from SuperMagnetMan) was seated into the pocket using epoxy, such that the magnetization (i.e., dipole axis) of the magnet is perpendicular to the central axis of the helix.

The helical swimmer fabricated herein is approximately the size that could be used in a number of in vivo medical applications [1]. However, we are primarily interested in applying our results to the type of microswimmers that have been designed for use as untethered micromanipulators under an optical microscope. In this regard, our helical swimmer is a scaled model of the microswimmers that have been created. We use corn syrup as our fluid medium herein, whereas microswimmers would typically swim in water or a fluid with properties similar to water. To

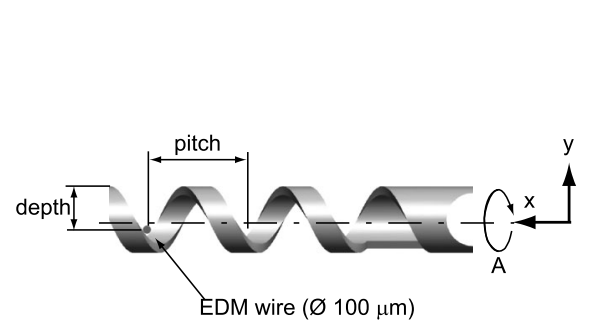

(a)

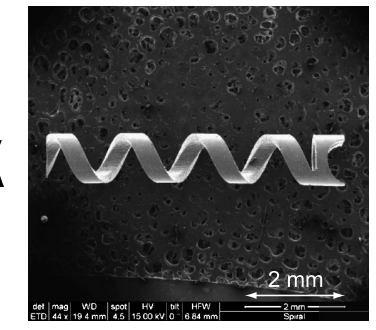

(b)

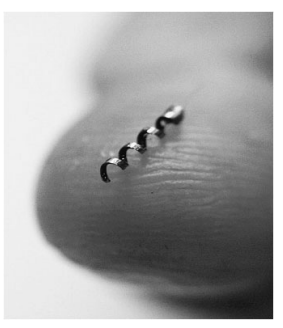

(c)

Figure 5. (a) Helical swimmer machined from 1-mm outer diameter Nitinol tubing using micro electrical discharge machining to produce a 5-mm long helix with 3.5 turns in a single sweep. (b) Scanning electron micrograph of the fabricated helix. (c) Helical swimmer on a human fingertip. 
understand for what size of microswimmer our results directly apply, we consider matching of Reynolds number:

$$
\mathrm{Re}=\frac{\rho_{\mathrm{mic}} L_{\mathrm{mic}} V_{\mathrm{mic}}}{\eta_{\mathrm{mic}}}=\frac{\rho_{\mathrm{mod}} L_{\mathrm{mod}} V_{\mathrm{mod}}}{\eta_{\mathrm{mod}}},
$$

where 'mod' denotes our model and 'mic' denotes the equivalent microscale swimmer in water. We are interested in finding the length scale of a microswimmer that corresponds to our experimental results. For our characteristic length we will consider the radius of the helix: $r_{\mathrm{h}}$. For our characteristic velocity we will consider the velocity of a segment of the helix moving through the fluid: $r_{\mathrm{h}} \Omega$. The achievable rotation speed $\Omega$ is invariant to scaling, due to the fact that magnetic torque scales with volume, which is proportional to length cubed, and rotational viscous drag also scales as length cubed (see model of Section 2). The result is a relation that describes the radius of the microswimmer that matches our experimental results:

$$
r_{\mathrm{h}, \text { mic }}=\sqrt{\left(\frac{\rho_{\text {mod }} \eta_{\text {mic }}}{\rho_{\text {mic }} \eta_{\text {mod }}}\right) r_{\mathrm{h}, \text { mod }}^{2}} .
$$

Our model swims in corn syrup, which has a viscosity of approximately $\eta_{\text {mod }}=$ $2500 \mathrm{cps}$, compared to $\eta_{\text {mic }}=1 \mathrm{cps}$ for water. The density of corn syrup is approximately $\rho_{\text {mod }}=1.36 \mathrm{~g} / \mathrm{ml}$, compared to $\rho_{\text {mic }}=1 \mathrm{~g} / \mathrm{ml}$ for water. The radius of our helical swimmer is $r_{\mathrm{h} \text {, mod }}=420 \mu \mathrm{m}$. Thus, the experimental results from our helical swimmer in corn syrup will correspond directly to a microswimmer with a radius $r_{\mathrm{h} \text {, mic }}=9.8 \mu \mathrm{m}$ swimming in water, which is reasonably close to the $1.5-\mu \mathrm{m}$ radius of the microswimmers of Ref. [4] and which places our results squarely into the low-Reynolds-number regime of interest.

\subsection{Characterization of the Magnetic Helical Swimmer}

Since (58) and (60) require only $d_{11}, d_{22}$ and $e_{11}$, only the two terms in ${ }^{\mathrm{h}} \mathbf{A}$ and the $b_{11}$ term from ${ }^{\mathrm{h}} \mathbf{B}$ need to be determined. Rather than explicitly calculating these parameters using the derived equations, (19)-(30), we experimentally measured the necessary parameters using a method similar to that described in Ref. [12] using (35) with known non-fluidic force (gravitational force in this case). The mass of the robot was measured to be $8.9 \mathrm{mg}$, resulting in a gravitational force acting on the swimmer of $-8.7 \times 10^{-5} \mathrm{~N}$. Using this force, the $a_{11}$ and $a_{22}$ terms of ${ }^{\mathrm{h}} \mathbf{A}$ were calculated by measuring the velocity of the swimmer as it sinks in the fluid at room temperature, first oriented vertically to obtain $a_{11}$ and then horizontally to obtain $a_{22}$. As the swimmer sinks, a static magnetic field was applied to enforce $\boldsymbol{\omega}=\mathbf{0}$, making (35) a relation between $\mathbf{f}$ and $\mathbf{v}$. With $\mathbf{v}$ measured and $\boldsymbol{\omega}=0, a_{11}=0.42 \mathrm{~N} \cdot \mathrm{s} / \mathrm{m}$ and $a_{22}=4.4 \mathrm{~N} \cdot \mathrm{s} / \mathrm{m}$ were found. To find the $b_{11}$ coefficient, rather than applying a static field to force $\boldsymbol{\omega}=\mathbf{0}$, the microswimmer was oriented vertically and made to rotate fast enough so the forward propulsion in the upward direction balanced the gravitational force downward. At this point of equilibrium, $\boldsymbol{\omega}$ is known and $\mathbf{v}=0$, turning (35) into a relation between applied force and rotation speed along the $\mathbf{x}_{\mathrm{h}}$ axis. Experimentally, the rotational 
speed necessary to balance the gravitational force was found to be $2.23 \mathrm{~Hz}$ making $b_{11}=-3.9 \times 10^{-5} \mathrm{~N} \cdot \mathrm{s}$. Since $d_{11}=1 / a_{11}, d_{22}=1 / a_{22}$ and $e_{11}=-b_{11} / a_{11}$, then we calculate the necessary parameters as: $d_{11}=2.4 \mathrm{~N}^{-1} \cdot \mathrm{m} / \mathrm{s}, d_{22}=0.23 \mathrm{~N}^{-1} \cdot \mathrm{m} / \mathrm{s}$ and $e_{11}=9.3 \times 10^{-5} \mathrm{~m}$.

\subsection{Experimental Results}

A variety of experiments were performed to study how the helical swimmer discussed in Sections 4.2 behaves under open-loop velocity control. Each experiment was performed with the swimmer submerged in a $50 \times 20 \times 20$-mm rectangular tank of corn syrup arranged in the center of the Helmholtz coils.

Presenting the user with control over the microswimmer's velocity in the world frame is an intuitive method to direct the swimmer. Without the algorithm presented in Section 3, performing simple maneuvers requires non-intuitive control over the direction to point the swimmer and how fast to rotate it. In the experiment shown in Fig. 6, the user held the vertical velocity at zero and manually adjusted the desired velocity in the horizontal direction to make the swimmer go from a stationary hover, ramp up to a constant horizontal velocity of $0.35 \mathrm{~mm} / \mathrm{s}$ and then return to hover. Although it is intuitive that the swimmer must turn in the direction of the desired velocity and increase its rotation speed, the exact pitch angle from verti$\mathrm{cal}$ and the rotation speed of the swimmer required to execute this maneuver are not immediately obvious to a human operator. With each change in input from the user, the control algorithm immediately adjusts the axis around which the magnetic field rotates and its rotational speed. The algorithm naively neglects the transient response of the swimmer to the changes in output. During the transient response, the robot sinks as seen in Fig. 6a between the swimmer at time 1 and 54 s. This is discussed further in Section 5.

The algorithm is not only designed to maintain a constant elevation by keeping $\left\|\mathbf{v}_{\text {ver }}\right\|=0$. Figure $7 \mathrm{a}$ and $\mathrm{b}$ shows the resulting trajectories after commanding the swimmer to attain a velocity of $0.1 \mathrm{~mm} / \mathrm{s}$ pitched $30^{\circ}$ above horizontal and $30^{\circ}$ below horizontal, respectively. The results show the swimmer moves in a straight trajectory approximately as commanded, achieving desired velocities of $0.12 \mathrm{~mm} / \mathrm{s}$ $20^{\circ}$ above horizontal and $0.17 \mathrm{~mm} / \mathrm{s} 17^{\circ}$ below the horizontal for the respective cases.

We found while performing the experiments that the steady-state behavior of the microswimmer is sensitive to changes in the viscosity of the corn syrup. The effect of fluid viscosity on fluidic characteristics can be understood by examining the terms of the ${ }^{\mathrm{h}} \mathbf{A}$ and ${ }^{\mathrm{h}} \mathbf{B}$ matrices. The $\xi_{\|}, \xi_{\perp}$ and $\xi_{\omega \mathrm{m}}$ coefficients that appear in each term of ${ }^{\mathrm{h}} \mathbf{A}$ and ${ }^{\mathrm{h}} \mathbf{B}$ are linearly scaled by the viscosity of the medium $(\eta)$ [15], making ${ }^{\mathrm{h}} \mathbf{A}$ and ${ }^{\mathrm{h}} \mathbf{B}$ themselves also linearly related to viscosity. The matrix ${ }^{\mathrm{h}} \mathbf{D}$ and ${ }^{\mathrm{h}} \mathbf{E}$ from which the control equations were derived are not related to viscosity in this manner, however. As ${ }^{\mathrm{h}} \mathbf{D}={ }^{\mathrm{h}} \mathbf{A}^{-1},{ }^{\mathrm{h}} \mathbf{D}$ (and subsequently its terms) is inversely proportional to viscosity. Analyzing the matrix ${ }^{\mathrm{h}} \mathbf{E}=-{ }^{\mathrm{h}} \mathbf{A}^{-1 \mathrm{~h}} \mathbf{B}$ shows that because ${ }^{\mathrm{h}} \mathbf{A}^{-1}$ is inversely proportional to viscosity and ${ }^{\mathrm{h}} \mathbf{B}$ is proportional to 


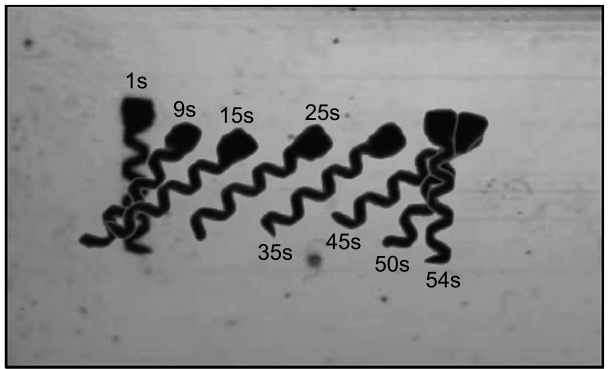

(a) Trajectory composition

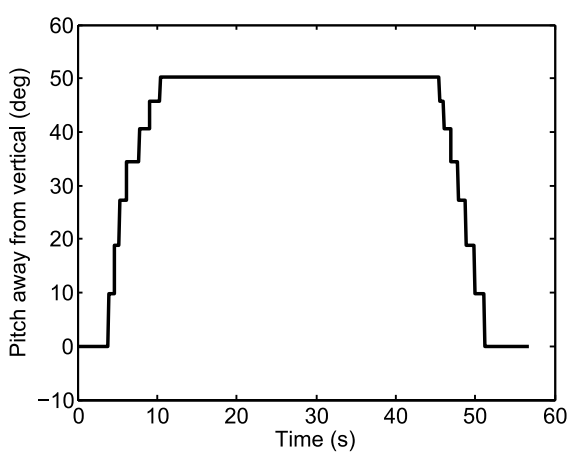

(c) Pitch angle vs. time

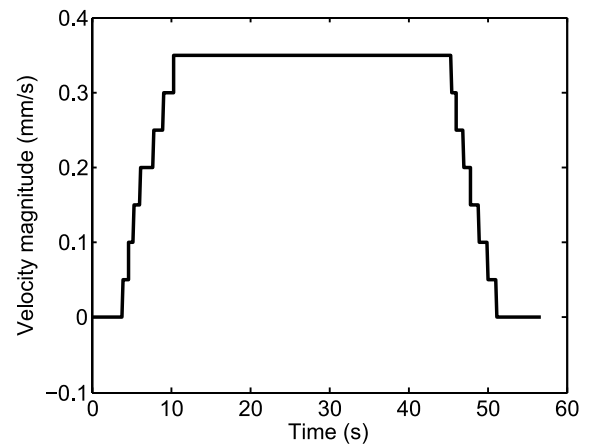

(b) Horizontal velocity vs. time

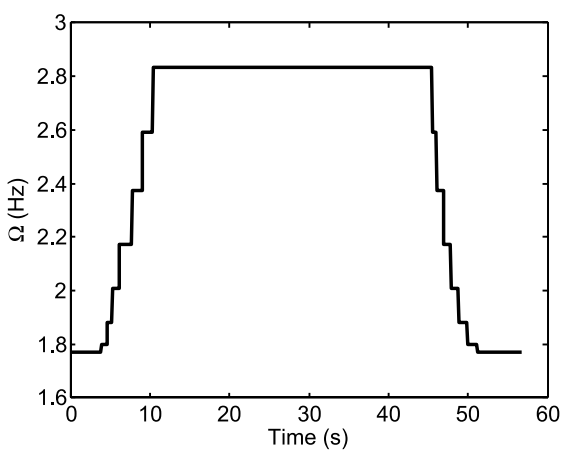

(d) Rotation speed $(\Omega)$ vs. time

Figure 6. Experimental results of a human operator commanding a hovering swimmer to accelerate up to a constant horizontal velocity and then decelerate back to hover while imparting no vertical motion. (a) Composite image sequence. (b) Manually commanded horizontal velocity. The human operator updates the desired velocity in $0.5-\mathrm{mm} / \mathrm{s}$ steps, up to a velocity of $0.35 \mathrm{~mm} / \mathrm{s}$. The outputs to control the swimmer — the pitch angle of $\tilde{\mathbf{x}}$ (c) and the rotation speed $\Omega$ (d) — are computed by the algorithm and are not intuitive to the human operator even for simple maneuvers such as this.

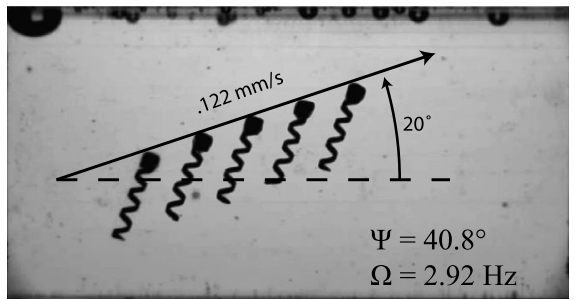

(a) Ascent under velocity control

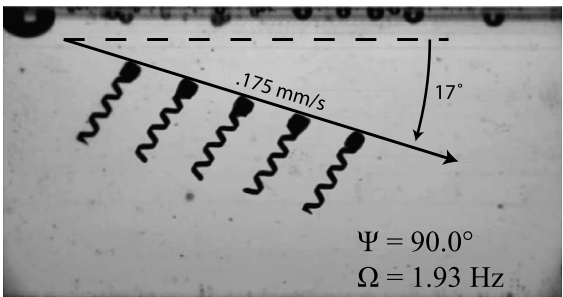

(b) Descent under velocity control

Figure 7. Composite experimental image sequences showing resulting trajectories after commanding the swimmer to attain a velocity of (a) $0.1 \mathrm{~mm} / \mathrm{s}$ angled $30^{\circ}$ above horizontal and (b) $0.1 \mathrm{~mm} / \mathrm{s}$ angled $30^{\circ}$ below horizontal. These results show a velocity error of $33 \%$ error in direction and $22 \%$ error in magnitude for (a). For (b), the results show a velocity error of $43 \%$ error in direction and $75 \%$ error in magnitude. Detailed discussion of these errors are presented in Section 4.4. 


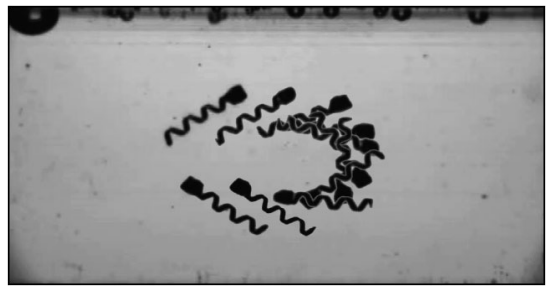

Figure 8. Composite experimental image sequence demonstrating that the algorithm seamlessly controls maneuvers such as this 'U-turn' while maintaining a constant forward velocity.

viscosity, their net effect cancels and ${ }^{\mathrm{h}} \mathbf{E}$ itself is invariant to changes in viscosity. The error shown in Fig. 7 is likely attributable to a change in fluid viscosity caused by fluctuation in ambient temperature after the swimming properties were characterized using methods discussed in the preceding section, as well as changes in the distance between the swimmer and the walls of the container. Simple sensitivity analysis when commanding the swimmer to obtain a velocity of $30^{\circ}$ above horizontal at $0.1 \mathrm{~mm} / \mathrm{s}$ (as is the case shown in Fig. 7a) shows that altering the parameters $d_{11}$ and $d_{22}$ (near the values obtained in Section 4.3) by overpredicting viscosity by $15 \%$ induces the microrobot to swim at an angle $10 \%$ less than desired above horizontal and with magnitude $17 \%$ less than desired. Underpredicting viscosity by $15 \%$ causes the microrobot to swim at an angle $10 \%$ more than desired above horizontal with magnitude $23 \%$ greater than desired. Additionally, we have found that the sensitivity to error in viscosity increases when operating in fluids with less viscosity than that used in this paper. Although potentially large, these disturbances can be compensated for by the human operator if he/she perceives that the swimmer is not moving as desired, since correction in the velocity commands are more intuitive to the human than corrections in pitch and rotation speed.

The algorithm presented in this paper is not limited to maneuvers where the swimmer is primarily oriented vertically, like those in the preceding experiments. The algorithm can determine the necessary control outputs given any spatial velocity. Figure 8 , for example, shows the trajectory of the swimmer under control of the algorithm for a maneuver where the user initially commands the swimmer to move horizontally and then rotates the velocity $180^{\circ}$ returning back to horizontal velocity opposite that at the start (as if performing a 'U-turn' in a car), while maintaining constant velocity magnitude. As can be seen in Fig. 8, the algorithm handles maneuvers such as these seamlessly.

\section{Discussion}

There are a number of assumptions built into the algorithm presented herein. (i) We assume that the microswimmer is always rotating in sync with the applied field, implying that the commanded rotation speed $\Omega$ is not above the step-out frequency of the microswimmer. The step-out frequency is well understood when swimming parallel to the central axis, but the effect of steering maneuvers on step-out is not 
well understood. (ii) We assume the microswimmer is swimming stably, meaning that, in addition to rotating in sync with the magnetic field, the central axis of the microswimmer $\mathbf{x}_{\mathrm{h}}$ is asymptotically converging on the field's rotation axis $\tilde{\mathbf{x}}$. During rapid steering maneuvers at high $\Omega$, microswimmers have been shown to go unstable, essentially requiring a kind of system reset. These first two assumptions are highly related. (iii) If the microswimmer's transient response to steering commands is too slow (i.e., the microswimmer's central axis $\mathbf{x}_{\mathbf{h}}$ takes too long to converge on $\tilde{\mathbf{x}}$ ), the microswimmer may ascend or descend unintentionally as it turns, resulting in an error in velocity until it reaches steady state. Using a smooth desired-velocity trajectory with limits on acceleration will mitigate unintended consequences of the microswimmer's transient behavior by ensuring that the microswimmer is never asked to make rapid changes in its pitch. This acceleration cap will also mitigate potential instability. (iv) We assumed a microswimmer with a spherical permanent magnet, but our fabricated swimmer, as well as previously published microswimmers, have different magnet geometries and some have utilized soft magnetic materials. With regard to magnet geometry, it is known that viscous drag coefficients are insensitive to small changes in geometry in Stokes flow. With regard to the use of soft magnetic material, this difference is inconsequential if we satisfy the above assumptions of stable swimming below step-out.

Since the presented algorithm is open loop and subject to modeling errors, it will be subject to errors and drift, but the drift should be slower than without the algorithm, providing the human operator with time to correct for perceived errors in the microswimmer's motion. In addition, the correction required by the operator will be straightforward compared to the prior method, since directly commanding velocity in the workspace is intuitive (e.g., if the microswimmer has undesired sinking, simply command it to move upward). It will also be straightforward to incorporate the algorithm presented herein into a closed-loop position or velocity control system using sensor feedback, such as the image from an optical microscope. This is likely to only require only the 3-d.o.f. position of the microswimmer or even just focus information. During the course of our experiments, we found that the performance of the algorithm is particularly sensitive to the viscosity of the fluid, which is affected by its temperature, as well as surface effects from the container, which are widely known to be important in a low-Reynolds-number regime. As discussed above, both of these factors could be accounted for in an intuitive manner by the human operator, but both factors also motivate the potential benefits of closing a control loop using visual feedback.

\section{Conclusions}

We have developed a 6-d.o.f. model for helical microswimmers in a low-Reynoldsnumber regime. Using this model, we derived a simple open-loop controller that allows a human user to directly command a desired velocity to magnetic helical microswimmers. The method incorporates a gravity-compensation routine that pre- 
vents the microswimmer from sinking due to its own weight. The method is more intuitive than controlling the orientation and rotation speed of the microswimmer directly, which is the method utilized in prior works. We found good agreement between experimental results and predictions.

\section{Acknowledgements}

This work was supported by the National Science Foundation under grants IIS0952718 and DGE-0654414. The Nitinol tube used to manufacture the helical microswimmer was provided by Johnson Matthey Medical. The photo in Fig. 5c is courtesy Utah Daily Chronicle. The authors wish to thank Lisandro Leon for his contributions during the construction of the Helmholtz coil system and Kathrin Peyer (ETH Zurich) for her extremely useful correspondence on RFT.

\section{References}

1. B. J. Nelson, I. K. Kaliakatsos and J. J. Abbott, Microrobots for minimally invasive medicine, Ann. Rev. Biomed. Eng. 12, 55-85 (2010).

2. C. Pawashe, S. Floyd and M. Sitti, Modeling and experimental characterization of an untethered magnetic micro-robot, Int. J. Robotics Res. 28, 1077-1094 (2009).

3. D. R. Frutiger, K. Vollmers, B. E. Kratochvil and B. J. Nelson, Small, fast, and under control: wireless resonant magnetic micro-agents, Int. J. Robotics Res. 29, 613-636 (2010).

4. L. Zhang, J. J. Abbott, L. X. Dong, B. E. Kratochvil, D. Bell and B. J. Nelson, Artificial bacterial flagella: fabrication and magnetic control, Appl. Phys. Lett. 94, 064107 (2009).

5. M. P. Kummer, J. J. Abbott, B. E. Kratochvil, R. Borer, A. Sengul and B. J. Nelson, OctoMag: an electromagnetic systems for 5-DOF wireless micromanipulation, in: Proc. IEEE Int. Conf. on Robotics and Automation, Anchorage, AK, pp. 1610-1616 (2010).

6. E. M. Purcell, Life at low Reynolds number, Am. J. Phys. 45, 3-11 (1977).

7. T. Honda, K. I. Arai and K. Ishiyama, Micro swimming mechanisms propelled by external magnetic fields, IEEE Trans. Magn. 32, 5085-5087 (1996).

8. A. Ghosh and P. Fischer, Controlled propulsion of artificial magnetic nanostructured propellers, Nano Lett. 9, 2243-2245 (2009).

9. J. J. Abbott, K. E. Peyer, M. Cosentino Lagomarsino, L. Zhang, L. X. Dong, I. K. Kaliakatsos and B. J. Nelson, How should microrobots swim? Int. J. Robotics Res. 28, 1434-1447 (2009).

10. K. E. Peyer, L. Zhang, B. E. Kratochvil and B. J. Nelson, Non-ideal swimming of artificial bacterial flagella near a surface, in: Proc. IEEE Int. Conf. Robotics and Automation, Anchorage, AK, pp. 96-101 (2010).

11. B. Behkem and M. Sitti, Design methodology for biomimetic propulsion of miniature swimming robots, ASME J. Dyn. Syst. Meas. Control 128, 36-43 (2006).

12. L. Zhang, J. J. Abbott, L. X. Dong, K. E. Peyer, B. E. Kratochvil, H. Zhang, C. Bergeles and B. J. Nelson, Characterizing the swimming properties of artificial bacterial flagella, Nano Lett. 9 , 3663-3667 (2009).

13. E. Lauga, W. DiLuzio, G. Whitesides and H. Stone, Swimming in circles: motion of bacteria near solid boundaries, Biophys. J. 90, 400-412 (2005).

14. J. Gray and G. J. Hancock, The propulsion of sea-urchin spermatozoa, J. Exp. Biol. 32, 802-814 (1955). 
15. J. Lighthill, Flagellar hydrodynamics, SIAM Rev. 18, 161-230 (1976).

16. J. J. Craig, Introduction to Robotics: Mechanics and Control, 2nd edn. Addison Wesley, Reading, MA (1989).

17. F. M. White, Viscous Fluid Flow, 2nd edn. McGraw-Hill, New York, NY (1991).

18. C. Orino, D. Rakwal, J. D. Jorgensen and E. Bamberg, Development of a precision, low-cost, small footprint wire electron discharge machine (WEDM), in: Proc. Amer. Society of Precision Engineering Conf., Monterey, CA, pp. 239-242 (2006).

\section{About the Authors}

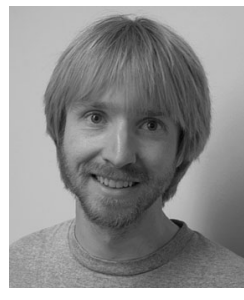

Arthur W. Mahoney received a BS degree in Computer Science and a BS degree in Computational Mathematics from Utah State University, Logan, UT, in 2009. As an undergraduate, he performed research and actively published in the fields of parallel computing, robot motion planning and computational biology. He is an NSF Graduate Research Fellow, a Hertz Fellowship finalist and is pursuing a PhD as a member of the Telerobotics Laboratory, Department of Mechanical Engineering, University of Utah, where he is studying magnetic control of mesoand microscale robots.

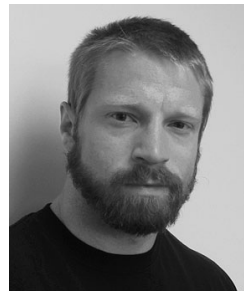

John C. Sarrazin received his MS and BS degrees in Mechanical Engineering from Montana State University, Bozeman, MT, in 2009 and 2007, respectively. He was awarded an IGERT Traineeship from the NSF for Biomimetic Robotics at the University of Utah, Salt Lake City, UT, in 2009, and is pursuing a $\mathrm{PhD}$ in Mechanical Engineering studying electroactive polymer actuators.

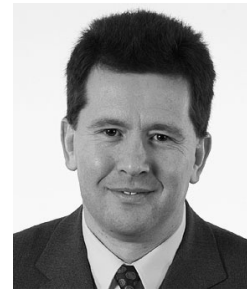

Eberhard Bamberg is currently a Research Assistant Professor in the Department of Mechanical Engineering at the University of Utah and the Director of the Precision Design Laboratory. He received a MS from Brunel University, Uxbridge, UK, in 1993, and a Dipl.-Ing. from the University of Stuttgart, Germany, in 1996, in Mechanical Engineering. He earned his $\mathrm{PhD}$ from the Massachusetts Institute of Technology, in 2000, in the area of precision design. His research interests include precision engineering, novel micro- and meso-level manufacturing technologies, non-traditional machining techniques for semiconductors, and optical instrumentation.

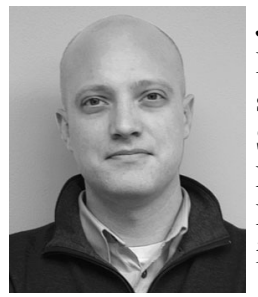

Jake J. Abbott received the PhD degree in Mechanical Engineering from Johns Hopkins University, Baltimore, MD, in 2005. He became a Postdoctoral Research Associate at the Institute of Robotics and Intelligent Systems, ETH Zurich, Switzerland, in 2005, and an Assistant Professor at the University of Utah, Salt Lake City, UT, in 2008. He is currently the Head of the Telerobotics Laboratory, Department of Mechanical Engineering, University of Utah, where his research involves medical and microscale telerobotics. 